\title{
Deciduosis of the Appendix Manifesting as Acute Abdomen in Pregnancy
}

\author{
Anita Balta, Maija Lubgane, Ineta Orube, Guna Ziemele, Andrejs Vanags, Janis Gardovskis, Ilze Strumfa \\ Riga Stradins University, Riga, Latvia
}

\section{SUMMARY}

Acute appendicitis is one of the most common surgical diseases during pregnancy. In rare cases, ectopic decidual tissues (deciduosis) can develop in the appendix and cause inflammation. Here we report a well-documented case of appendicular deciduosis presenting with acute abdominal pain and resulting in gangrenous appendicitis.

Key words: appendicitis, pregnancy, deciduosis

\section{AIM OF THE DEMONSTRATION}

In order to increase the awareness of rare appendicular diseases and the peculiar differential diagnosis of appendicitis in pregnancy, here we present a rare case of appendicular deciduosis causing acute appendicitis in a pregnant lady.

\section{CASE REPORT}

A 33-year-old primigravida woman entered the hospital in 28/29 weeks of gestation. The patient reported right lower abdominal pain lasting for 11 hours. Initially, preterm delivery was suspected. The body temperature was $37.2 \mathrm{C}^{0}$. The white blood cell count was $16.7 \times 10^{9}$ /L (laboratory reference interval (LRI) $4-10$ ). The level of C-reactive protein (CRP) reached $27.4 \mathrm{mg} / \mathrm{L}$ (LRI 0 5). The abdominal ultrasonography showed picture of acute appendicitis as the appendix had thickened wall and was surrounded by a small amount of liquid. Gross intraoperative findings were suggestive of gangrenous appendicitis. Conventional appendectomy was performed. The postoperative period was uneventful. The patient received analgetic and antibiotic therapy. Fetal movements were monitored and sensed well. Uterus was normotonic. There was no abdominal pain except sensitivity around the surgical wound by palpation. By pathologic examination, the removed appendix grossly measured $6 \times 1.2 \times 0.8 \mathrm{~cm}$ and showed uneven surface. Microscopically, gangrenous appendicitis was revealed along with perforation and wide areas of deciduous ectopic reaction s. deciduosis (Fig.1), characterised by nodules of discohesive large polygonal cells with widespread degenerative cytoplasmic vacuolisation resulting in signet ring cell like appearance. Acute inflammation with fibrinous and purulent component extended to the periapendicular and mesenteriolar tissues. By immunohistochemical investigation (IHC), the large polygonal and vacuolated cells in pathologic foci expressed progesterone receptors and vimentin but lacked pan-cytokeratin and calretinin. Thus, IHC confirmed deciduosis and ruled out malignant tumour. The differential diagnosis over the whole course of illness comprised acute appendicitis, preterm delivery, cancer, acute pyelonephritis or rupture of ovarian cyst. However, considering the intraoperative surgical findings as well as morphological and IHC data, the final diagnosis was appendicular deciduosis, complicated by gangrenous appendicitis and phlegmonous periappendicitis and mesenteriolitis.

\section{DISCUSSION}

Development of decidual cells outside the endometrium, first described by Walker in 1887, is named ectopic decidua or deciduosis (Walker, 1887; Bolat et al., 2012). Such changes most commonly affect ovaries, uterine serosa (Kondoh et al., 2012), fallopian tubes and cervix (Bolat et al., 2012). Deciduosis is less frequently seen in the appendix (Adhikari and Shen, 2013), diaphragm, liver, spleen, paraaortic and pelvic lymph nodes or renal pelvis (Bolat et al., 2012). The involvement of omentum is considered rare by some authors (Adhikari and Shen, 2013), while others suggest that it could be disclosed frequently, if careful sampling would be possible (Buttner et al., 1993; Rodriguez et al., 2006). Deciduosis can be found incidentally in tissues removed or biopsied during a caesarean section, treatment of tubal pregnancy, elective tubal ligation and appendectomy (Bolat et al., 2012).

The pathogenesis of deciduosis is not yet fully understood (Kondoh et al., 2012) and the physiologic nature of this reaction is considered controversial (Bolat et al., 2012). The most frequently suggested explanations include de novo development from submesothelial stroma, or decidual transformation of pre-existing endometriosis (Bolat et al., 2012; Kondoh et al., 2012; Adhikari and Shen, 2013). The de novo pathway would involve progesterone-related subserosal stromal metaplasia. Confirming the role of hormonal influences, deciduosis regresses within $4-6$ weeks after pregnancy along with decidual involution (Bolat et al., 2012). High level of progesterone in twin gestations has been attributed to diffuse peritoneal deciduosis in such patients (Adhikari and Shen, 2013). Occasionally, deciduosis in non-pregnant ladies has been explained by adrenal progesterone secretion acting on submesothelial 
stroma sensitized by inflammation or trauma (Buttner et al., 1993; Rodriguez et al., 2006). Although deciduosis has been identified in patients lacking any history of endometriosis (Bolat et al., 2012), increased awareness of this condition is recommended managing pregnancy in women with history of dysmenorrhea or endometriosis (Kondoh et al., 2012).

Albeit deciduosis frequently lacks clinical symptoms, it can manifest by abdominal pain and leukocytosis that has been related to the production of granulocyte colony-stimulating factor (Kondoh et al., 2012). Occasionally, intestinal obstruction (Rodriguez et al., 2006) or significant abdominal or gastrointestinal haemorrhage can develop (Bashir et al., 1995; Kondoh et al., 2012). Lethal outcome of deciduosis has been previously reported underlining the necessity of correct diagnosis and timely surgical treatment (Theissig et al., 1988).

In contrast to frequently mentioned asymptomatic course, our patient had pain and leukocytosis. In the presented case, these manifestations can be explained by severe acute inflammation related to rich presence of neutrophilic leukocytes. The gangrenous tissues were widely haemorrhagic. However, there was no evidence of prior primary haemorrhage within the tissues.

The clinical diagnosis of deciduosis is difficult even in the presence of widespread intraabdominal involvement (Kondoh et al., 2012). The intraoperative findings can yield broad differential diagnosis including primary or metastatic malignant tumours as deciduoid mesothelioma, abdominal carcinomatosis or metastatic abdominal spread of malignant melanoma (Ustun et al., 2011; Adhikari and Shen, 2013). Regarding the differential diagnosis with malignant tumours, morphological examination of the removed tissues is the "gold standard". Deciduosis is characterised by nodular architecture. The nodules are composed of large cells with well-defined cell borders and eosinophilic cytoplasm. Degenerative changes lead to cytoplasmic vacuolisation, physaliphorous, signet ring or lipoblastic appearance of cells and myxoid stroma. Mitotic activity and/ or nuclear atypia are absent (Rodriguez et al., 2006; Bolat et al., 2012). However, even by light microscopy decidual change may be mistaken for metastatic carcinoma or malignant mesothelioma (Adhikari and Shen, 2013). In such cases, IHC is advisable. Cytoplasmic positivity of vimentin and nuclear presence of progesterone receptors along with lack of pan-cytokeratin, HMB-45 and calretinin is characteristic for deciduosis. Although the lesions typically are negative for cytokeratin, few positive cells can be present (Bolat et al., 2012; Adhikari and Shen, 2013). Metastatic carcinoma is characterised by cytokeratin expression, while mesothelioma - by calretinin along with pan-cytokeratin, cytokeratin 5/6 and vimentin. HMB-45 expression is almost diagnostic of melanoma (Ustun et al., 2011; Bolat et al., 2012; Adhikari and Shen, 2013).

Appendectomy is the most common non-obstetric surgical intervention in pregnant patients. The incidence of acute appendicitis in pregnancy is estimated as $0.05-$
0.13\% (Kirshtein et al., 2009; Chung et al., 2013). Thus, appendicitis must be considered as the cause of acute abdomen during pregnancy. Cases of deciduosis manifesting by abdominal pain, leukocytosis and elevated CRP level necessitate even more careful consideration of appendicitis in the differential diagnosis (Kondoh et al., 2012). The history of the described case shows an additional novel, clinically highly important fact - the appendicitis can also be true complication of deciduosis

In conclusion, deciduosis is a rare, pregnancy-related process that in rare cases can affect appendix. In our patient, gangrenous inflammation supervened necessitating urgent appendectomy. Thus, deciduosis should be considered in the differential diagnosis of acute abdomen in pregnancy.

\section{Conflict of interest: None}

\section{REFERENCES}

1. Adhikari LJ, Shen R. Florid diffuse peritoneal deciduosis mimicking carcinomatosis in a primigravida patient: a case report and review of the literature // Int J Clin Exp Pathol, 2013; 6: $2615-2619$

2. Bashir RM, Motgomery EA, Gupta PK, Nauta RM, Crockett SA, Collea JV, al-Kawas FH. Massive gastrointestinal hemorrhage during pregnancy caused by ectopic decidua of the terminal ileum and colon // Am J Gastroenterol, 1995; 90:1325 - 1327

3. Bolat F, Canpolat T, Tarim E. Pregnancyrelated peritoneal ectopic decidua (deciduosis): morphological and clinical evaluation // Turk Patoloji Derg, 2012; 20:56 - 60

4. Buttner A, Bassler R, Theele C. Pregnancyassociated ectopic decidua (deciduosis) of the greater omentum. An analysis of 60 biopsies with cases of fibrosing deciduosis and leiomyomatosis peritonealis disseminata // Pathol Res Pract, 1993; $189: 352-359$

5. Chung JC, Cho GS, Shin EJ, Kim HC, Song OP. Clinical outcomes compared between laparoscopic and open appendectomy in pregnant women // Can J Surg, 2013; 56:341 - 346

6. Kirshtein B, Perry ZH, Avinoach E, Mizrahi S, Lantsberg L. Safety of laparoscopic appendectomy during pregnancy // World J Surg, 2009; 33: $475-480$

7. Kondoh E, Shimizu M, Kakui K, Mikami Y, Tatsumi $\mathrm{K}$, Konishi I. Deciduosis can cause remarkable leukocytosis and obscure abdominal pain // J Obstet Gynaecol Res, 2012; 38:1376 - 1378

8. Theissig F, Kemmer C, Kunze KD. Fatal course of mesenteric deciduosis in a gravida I // Pathologe, 1988; 9:50 - 54

9. Ustun H, Astarci HM, Sungu N, Ozdemir A, Ekinci C. Primary malignant deciduoid peritoneal mesothelioma: a report of the cytohistological and immunohistochemical appearances // Diagn Cytopathol, 2011; 39:402-408 
10. Walker A. Der bau der eihaeute bei graviditatis abdominalis // Virchows Arch Path Anat, 1887; 197:72-99

\section{Address:}

Andrejs Vanags

Department of Surgery,

Riga Stradins University,

Pilsonu Street 13, LV1002, Riga, Latvia

E-mail: Andrejs.Vanags@rsu.lv

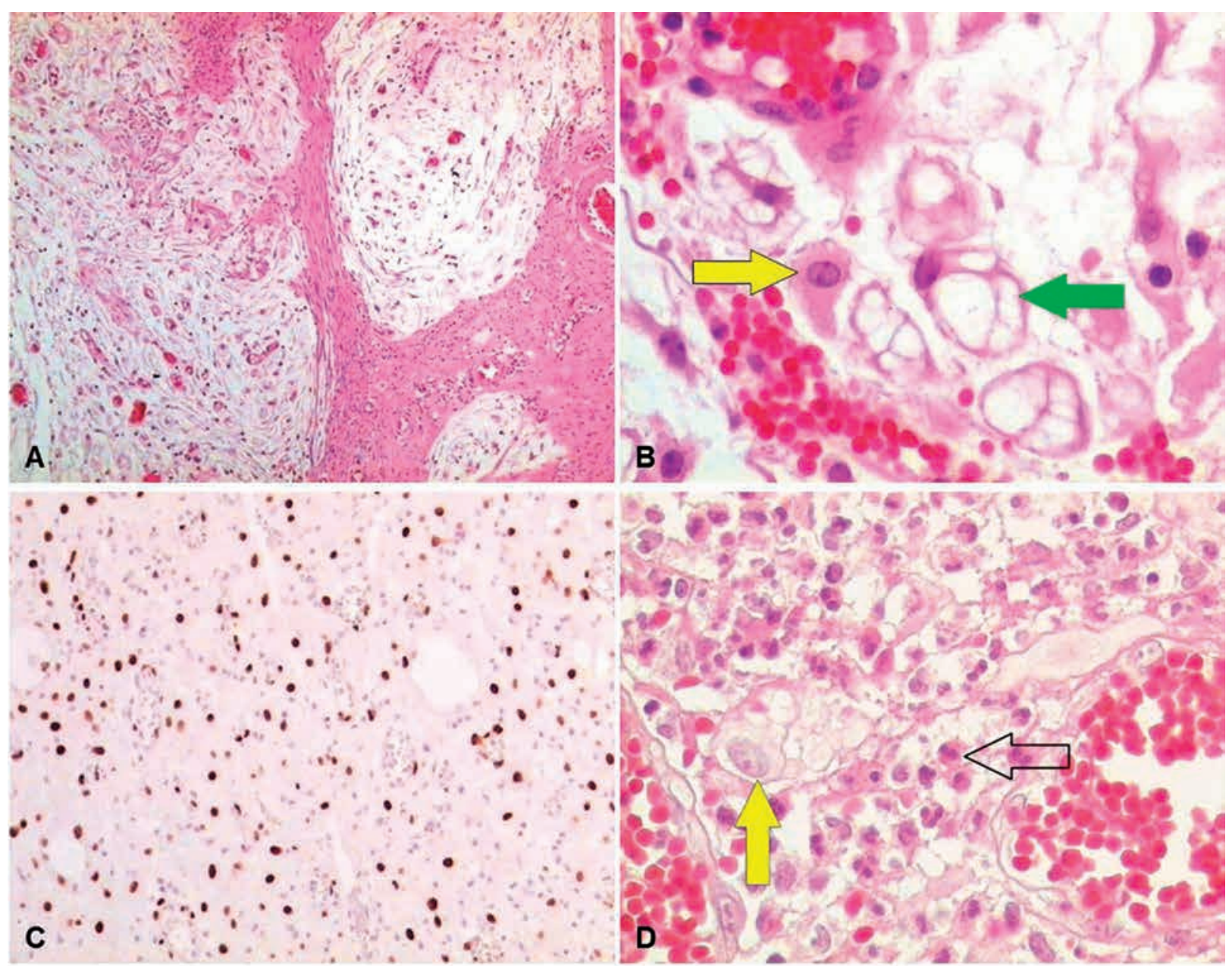

Fig. 1. Appendicular deciduosis. A, Overview of the affected tissue. Note the nodular foci of deciduosis. Haematoxylin-eosin (HE), original magnification (OM) 50x. B, Cell morphology of the decidual foci. Note the intact deciduoid cell (yellow arrow) and the degenerative changes resulting in signet ring cell like appearance (green arrow). HE, OM 400x. C, Expression of progesterone receptors (PR) in deciduoid cells. Immunoperoxidase, anti-PR, OM 100x. D. Purulent inflammation (arrow) surrounding a deciduoid cell (yellow arrow). HE, OM 400x. 